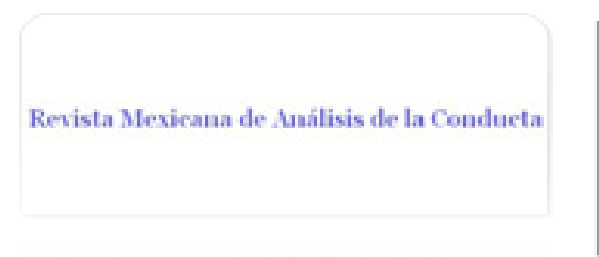

Revista Mexicana de Análisis de la Conducta ISSN: 0185-4534

editora@rmac-mx.org

Sociedad Mexicana de Análisis de la Conducta México

Flores, Carlos; Bruner, Carlos

Efectos de diferentes probabilidades de reforzamiento sobre la precisión de palomas en tareas de igualación a la muestra: alargando la duración del intervalo entre reforzadores Revista Mexicana de Análisis de la Conducta, vol. 33, núm. 1, junio, 2007, pp. 31-41

Sociedad Mexicana de Análisis de la Conducta

Guadalajara, México

Disponible en: http://www.redalyc.org/articulo.oa?id=59333103

- Cómo citar el artículo

- Número completo

- Más información del artículo

- Página de la revista en redalyc.org

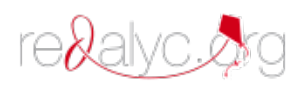

Sistema de Información Científica Red de Revistas Científicas de América Latina, el Caribe, España y Portugal Proyecto académico sin fines de lucro, desarrollado bajo la iniciativa de acceso abierto 


\section{EFECTOS DE DIFERENTES PROBABILIDADES DE REFORZAMIENTO SOBRE LA PRECISIÓN DE PALOMAS EN TAREAS DE IGUALACIÓN A LA MUESTRA: ALARGANDO LA DURACIÓN DEL INTERVALO ENTRE REFORZADORES}

EFFECTS OF DIFFERENT PROBABILITIES OF REINFORCEMENT ON ACCURACY IN A MATCHING TO SAMPLE TASK: INCREASING THE INTEREINFORCEMENT INTERVAL

\section{CARLOS FLORES* Y CARLOS BRUNER**}

*UNIVERSIDAD DE GUADALAJARA-CENTRO DE ESTUDIOS

E INVESTIGACIONES EN COMPORTAMIENTO

**UNIVERSIDAD NACIONAL AUTÓNOMA DE MÉXICO-FACULTAD

DE PSICOLOGÍA

\section{RESUMEN}

Se evaluaron los efectos de alargar la duración del intervalo entre reforzadores mediante la reducción de la probabilidad de reforzamiento sobre la precisión de palomas en tareas de igualación a la muestra. Para cada tres palomas se programó un valor de probabilidad de reforzamiento $(1.0,0.5$, 0.25 y 0.12 ) que resultó en diferentes duraciones del intervalo entre reforzadores $(19,38,76$ y 152 s). La velocidad de adquisición fue más rápida y el índice de discriminación fue más alto para las palomas expuestas al intervalo entre reforzadores más largo. Se identifica al intervalo entre reforzadores como una variable más general que controla la precisión y la adquisición de discriminaciones condicionales.

Palabras clave: Igualación a la muestra, palomas, intervalo entre reforzadores, probabilidad de reforzamiento.

1. La correspondencia relacionada con este trabajo puede enviarse al primer autor a Centro de Estudios e Investigaciones en Comportamiento, Francisco de Quevedo No. 180, Col. Arcos Vallarta, Guadalajara Jalisco México, e-mail: cjflores@servidor.unam.mx

2. Comisión del primer autor por la Universidad Nacional Autónoma de México, Facultad de Estudios Superiores Iztacala. 


\begin{abstract}
Using a matching to sample task pigeons were exposed to different durations of interreinforcer interval $(19,38,76$ and $152 \mathrm{~s})$ according to different probability of reinforcement values $(1.0,0.5,0.25,0.12)$. The speed of acquisition was quicker and the discrimination index was higher for the pigeons exposed to a longer interreinforcer interval. It is identified to the interreinforcer interval like a more general variable that controls the accuracy levels and the acquisition speed of conditional discriminations.

Key words: Matching to sample, pigeons, interreinforcer interval, probability of reinforcement.

En el campo de la discriminación condicional y utilizando tareas de igualación a la muestra se han estudiado los efectos de dos variables temporales: la duración del estímulo muestra (EM) y la duración del intervalo entre ensayos (IEE), aumentar la duración de cualquiera de estas dos variables resulta en una más rápida adquisición y en más altos índices de discriminación (Holt \& Shafer, 1973; Maki, Moe \& Bierley, 1977; Nelson \& Wasserman, 1978; Roberts, 1972, 1980; Roberts \& Grant, 1974; Roberts \& Kraemer, 1982).

En un estudio reciente, Flores y Bruner (2005) demostraron que no son la duración del EM ni la del IEE las variables que controlan la precisión del responder en tareas de igualación a la muestra y que una mayor duración del intervalo entre reforzadores (IER) resulta en más altos niveles de precisión de la respuesta. La estrategia que siguieron Flores y Bruner para evaluar los efectos del IER resultó de la combinación de diferentes duraciones tanto del EM como del IEE.

En su estudio, Flores y Bruner entrenaron a cuatro grupos de palomas en una tarea de igualación de la muestra con diferentes duraciones del IER (36 s y 126 s), para dos grupos de palomas el IER (36 s) resultó de la combinación de diferentes duraciones del EM ( 3 s y 30 s) y del IEE (30 s y 3 ) manteniendo la duración del estímulo de comparación (ECO) en $3 \mathrm{~s}$; mientras que para otros dos grupos de palomas el IER (126 s) fue el resultado de utilizar diferentes duraciones del EM (120 s y 3 s) y del IEE (3 s y 120 s). De las diferentes duraciones del EM y del IEE resultó la posibilidad de igualar la duración del IER en 36 s o 126 s pero variando los valores absolutos (EM+ECO+IEE) tanto del EM como del IEE.

Sin embargo, variar la duración del IER también puede ser resultado de la manipulación sistemática de los valores de la probabilidad de reforzamiento por responder al ECO correcto. De hecho, reducir la probabilidad de reforzamiento por responder al ECO correcto implica alargar la duración del intervalo promedio entre reforzadores.
\end{abstract}


En algunos estudios se ha reportado que la variación en la probabilidad de reforzamiento mejora la precisión del responder (e.g., De Long \& Wasserman, 1981; Santi \& Roberts, 1985a, 1985 b; Urcuioli, 1990a, 1990b).

Empleando un procedimiento de igualación de la muestra, De Long y Waserman (1981) reforzaron las respuestas ante el ECO1 dado el EM1 con una probabilidad de 1.0, mientras que las respuestas al ECO2 dado el EM2 se reforzaron con una probabilidad de 0.2 (grupo con procedimiento de consecuencias diferenciales); para otro grupo (procedimiento con consecuencias iguales o no diferenciales) tanto las respuestas al ECO1 dado el EM 1 como las respuestas al ECO2 dado el EM2 se reforzaron con una probabilidad de 0.6 (para una revisión de los procedimientos con consecuencias diferenciales ver Goeters, Blakely \& Poling, 1992). De Long y Waserman (1981) reportaron que la precisión del responder fue más alta para el grupo con consecuencias diferenciales comparado con el grupo en las que las respuestas correctas siempre se reforzaron con una probabilidad de 0.6

Recientemente, Brown y White (2005) evaluaron los efectos de señalar la probabilidad de reforzamiento en una tarea de igualación a la muestra demorada. En la mitad de los ensayos programados en cada sesión se presentaba una señal correlacionada con una probabilidad de reforzamiento de 1.0 , mientras que en la otra mitad de los ensayos se presentaba una señal correlacionada con una probabilidad de 0.2. Reportaron que la precisión del responder fue más alta en los ensayos en los que la respuesta se reforzó con una probabilidad de 1.0 .

Como se puede observar, la manipulación de la probabilidad de reforzamiento es una variable que se ha explorado empleando tareas de igualación a la muestra, sin embargo, estos estudios no fueron diseñados para evaluar explícitamente el efecto de la duración del IER sobre la velocidad de adquisición y la precisión del responder. El presente estudio se diseñó con el propósito de evaluar sistemáticamente los efectos de diferentes duraciones del intervalo entre reforzadores sobre la velocidad de adquisición y el índice de precisión del responder y de extender la generalidad de los resultados reportados por Flores y Bruner (2005). Extender la generalidad del hallazgo permitiría dar fuerza a la afirmación de que no es la mayor duración del EM ni la del IEE las variables responsables de observar índices de precisión más elevados y mayores tasas de adquisición en tareas de igualación de la muestra. Específicamente se alargó la duración del intervalo entre reforzadores mediante la reducción de la probabilidad de reforzamiento por responder al ECO correcto. 


\section{MÉTODO}

\section{Sujetos}

Se utilizaron 12 palomas de seis meses de edad experimentalmente ingenuas al inicio de la investigación. Se alojó a las palomas en jaulas individuales con acceso libre al agua y acceso restringido a la comida para mantenerlas al $80 \%$ de su peso en alimentación libre durante todo el experimento.

\section{Aparatos}

Dos cámaras experimentales Coulburn Instruments $(31 \mathrm{~cm}$ de largo, $30.5 \mathrm{~cm}$ de altura y $25.5 \mathrm{~cm}$ de ancho) para palomas. Tres teclas translúcidas de 2.5 $\mathrm{cm}$ de diámetro, que proyectaron luces de diferente color (rojo y verde) y requirieron de una fuerza de $0.15 \mathrm{~N}$ para ser operadas, se montaron alineadas horizontalmente en el panel frontal de cada cámara a $21 \mathrm{~cm}$ del piso y separadas entre sí por una distancia de $6 \mathrm{~cm}$. Una apertura de $5 \mathrm{~cm}$ de largo x 5 $\mathrm{cm}$ de ancho ubicada a $4 \mathrm{~cm}$ del piso del panel frontal de la cámara permitía el acceso al alimento. Cada presentación del alimento tuvo una duración de 3 s y se acompañó por la iluminación de una luz blanca de 5 w. Los eventos experimentales y la recolección de datos se llevaron a cabo con la ayuda de una computadora (PC-486) conectada a una interfase (Med Associates). Las cámaras experimentales se colocaron en cajas a prueba de ruidos (Coulbourn Instruments E10-10) y se ubicaron en un cuarto adyacente al cubículo de trabajo.

\section{Procedimiento}

La respuesta de picar las teclas se estableció mediante un procedimiento de automoldeamiento (Brown \& Jenkins, 1968) en el cual la tecla central se iluminaba en color rojo o verde durante $8 \mathrm{~s}$ y a su terminación se activaba el dispensador de alimento durante $4 \mathrm{~s}$; al término de este periodo iniciaba un IEE de 60 s durante el cual se elegía al azar el color de la tecla (rojo o verde) para el siguiente ensayo de entrenamiento. En cualquier momento de los $8 \mathrm{~s}$ de la iluminación de la tecla, la respuesta de las palomas cancelaba la señal y producía la activación del dispensador de alimento durante $4 \mathrm{~s}$. En cada sesión se programaron 60 ensayos y el entrenamiento concluyó cuando las palomas respondieron a la tecla en el $50 \%$ de los ensayos programados. Después de esto el procedimiento de igualación a la muestra inició formalmente. Las sesiones iniciaban con el EM que consistió en la iluminación de color rojo o verde de la tecla central por una duración de $4 \mathrm{~s}$. La terminación del EM coincidió con la iluminación de las teclas laterales, una en color rojo y la otra en color verde, que sirvieron como ECO. Si las palomas picaban la tecla iluminada de diferente color al que había servido como el EM, las luces de las teclas laterales se apagaban y las palomas tenían acceso al alimento 
durante $3 \mathrm{~s}$ (respuesta correcta o acierto). Si las palomas picaban la tecla iluminada con un color igual al que tuvo en ese ensayo el EM se apagaban las teclas de comparación y se iniciaba un tiempo fuera de $3 \mathrm{~s}$ (respuesta incorrecta o error). El IEE (12 s) iniciaba inmediatamente después del tiempo fuera o del acceso al alimento, lo que hubiese ocurrido primero. Para todas las palomas la duración del ECO fue de $3 \mathrm{~s}$. Todas las palomas estuvieron en estas condiciones durante 40 sesiones y cada sesión concluyó cuando se completaron 30 reforzadores.

Para cada tres palomas se utilizó un valor diferente de probabilidad de reforzamiento por responder al ECO correcto. Los valores de probabilidad de reforzamiento $(1.0,0.5,0.25$ y 0.125$)$ dieron lugar a diferentes duraciones del IER, siendo iguales a 19, 38, 76, y $152 \mathrm{~s}$. En la Tabla 1 se muestran las duraciones del EM, ECO y del IEE en cada una de las condiciones.

\begin{tabular}{|c|c|c|c|c|}
\hline PER & IEE & EM & ECO & IER \\
\hline 1.0 & 12 & 4 & 3 & 19 \\
\hline 0.50 & 12 & 4 & 3 & 38 \\
\hline 0.25 & 12 & 4 & 3 & 76 \\
\hline 0.12 & 12 & 4 & 3 & 152 \\
\hline
\end{tabular}

Tabla 1. Duraciones de los diferentes elementos de la tarea experimental. IEE = intervalo entre ensayos, EM= estímulo muestra, $E C O=$ estímulos de comparación, IER= intervalo entre reforzadores, $P E R=$ probabilidad de reforzamiento.

\section{RESULTADOS}

El dato de interés fue el índice de precisión (IP) que se calculó como sigue:

$$
I P=A / A+E(1)
$$

donde $\mathrm{A}$ representa el número total de aciertos por sesión y $\mathrm{E}$ representa el número total de errores por sesión.

El índice de precisión promedio de los tres sujetos de cada condición se presenta en la Figura 1. En esta figura se puede observar que los índices de precisión incrementaron en función de una mayor duración del intervalo entre reforzadores, con el IER de $152 \mathrm{~s}$ se encontraron índices de precisión más elevados que con IER de 76, 38 y $19 \mathrm{~s}$. De hecho, se puede observar un ordenamiento en el índice de precisión, una mayor duración del intervalo entre reforzadores resultó en índices de precisión más elevados. 
Con fines de análisis de las condiciones de adquisición iniciales y terminales, se presentarán por separado datos de las primeras 20 sesiones y de las últimas 20 sesiones experimentales.

Los índices de precisión promedio de las primeras 20 sesiones fueron 0.39 y 0.49 para los sujetos con IER de $19 \mathrm{~s}$ y $38 \mathrm{~s}$, respectivamente; mientras que para los sujetos con IER de $76 \mathrm{~s}$ y $152 \mathrm{~s}$ los índices de precisión fueron 0.53 y 0.59 , respectivamente. Un ANOVA mostró diferencias significativas entre los índices de precisión de las palomas de cada condición $\mathrm{F}$ $(3,8)=116.045, p<0.001$. Comparaciones múltiples (Tukey) revelaron que el índice de precisión entre cada una de las cuatro condiciones fue significativamente diferente.

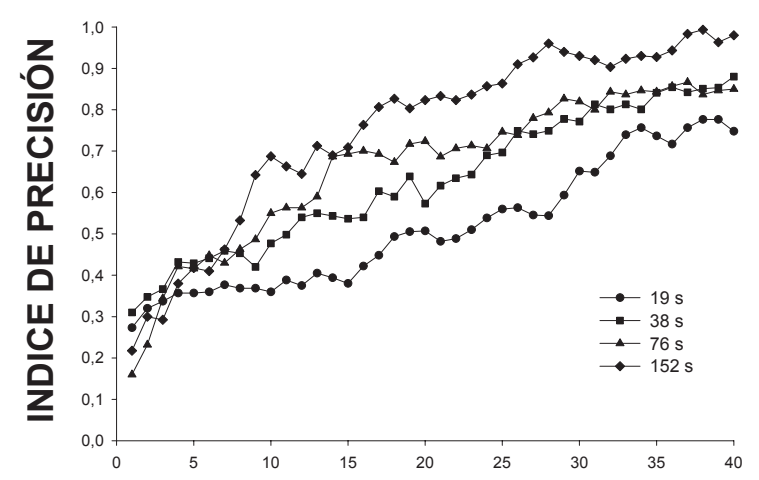

SESIONES

Figura 1. Índice de precisión promedio de los tres sujetos de cada condición.

Los índices de precisión promedio de las últimas 20 sesiones fueron 0.64 y 0.77 para los sujetos con IER de $19 \mathrm{~s}$ y $38 \mathrm{~s}$, respectivamente; mientras que para los sujetos con IER iguales a 76 s y 152 s fueron 0.80 y 0.91 , respectivamente. Un ANOVA de las últimas 20 sesiones mostró diferencias entre los índices de precisión de las cuatro condiciones $F(3,8)=201.333, p<0.001$. Un análisis de comparaciones múltiples mostró que no se encontraron diferencias significativas entre los índices de precisión de los sujetos con IER de $38 \mathrm{~s}$ y de $76 \mathrm{~s}$. El resto de las comparaciones fueron significativas, los sujetos 


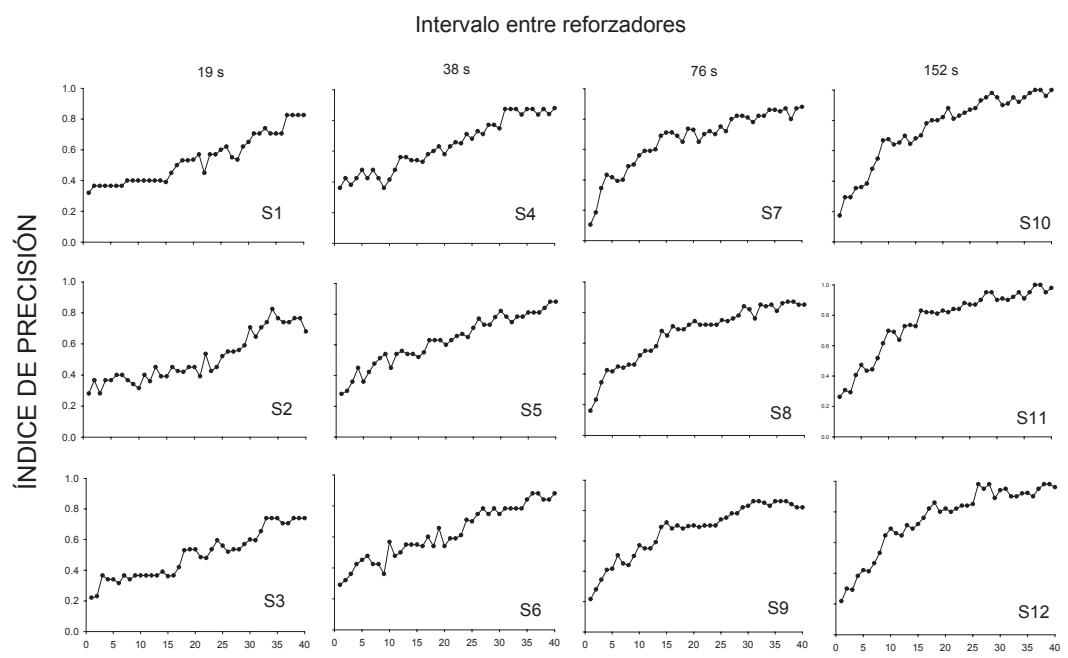

SESIONES

Figura 2. Índice de precisión por sesión de cada sujeto en cada condición.

con un IER de 152 s tuvieron índices de precisión significativamente más elevados que en el resto de condiciones, los sujetos con un IER de $19 \mathrm{~s}$ fueron los que tuvieron los índices de precisión más bajos.

En la Figura 2 se presenta el índice de precisión por sesión de cada sujeto en cada condición experimental. Esta figura muestra que los sujetos de las condiciones con IER de $19 \mathrm{~s}$ y de $38 \mathrm{~s}$ de duración requirieron hasta 35 sesiones para alcanzar índices de precisión iguales o superiores a 0.8 , mientras que los sujetos de las condiciones con IER de 76 s y 152 s requirieron entre 20 y 25 sesiones para llegar a índices de precisión superiores a 0.8. Este resultado destaca que los sujetos con mayores IER tienen tasas de adquisición más rápidas que los sujetos con las menores duraciones del IER.

\section{DISCUSIÓN}

El propósito del presente estudio fue evaluar el efecto de alargar la duración del intervalo entre reforzadores mediante la reducción de la probabilidad de reforzamiento sobre la adquisición y la precisión del responder en tareas de igualación a la muestra. Los resultados del estudio son consistentes con los reportados 
por Flores y Bruner (2005) al observar que una mayor duración del intervalo entre reforzadores resultó en índices de precisión más elevados. Con base en los resultados reportados en varios estudios (Holt \& Shafer, 1973; Maki, Moe \& Bierley, 1977; Nelson \& Wasserman, 1978; Roberts, 1972, 1980; Roberts \& Grant, 1974; Roberts \& Kraemer, 1982), en el trabajo de Flores y Bruner se podían predecir índices de precisión más elevados con las duraciones más largas del EM (120 s o 30 s), pero también se podían esperar índices más elevados con los IEE de mayor duración (120 s o 30 s), sin embargo esta predicción no fue confirmada. No se encontraron diferencias entre los índices de precisión de los dos grupos en los que se igualó el IER a $36 \mathrm{~s}$ a pesar de que en uno de ellos la duración del EM fue mucho mayor que en la del otro y de que la duración del IEE también fuera mayor; tampoco se encontraron diferencias entre los índices de los dos grupos con IER de 126 s, pero si se encontraron índices considerablemente más elevados y tasas de adquisición más rápidas en los grupos con IER de $126 \mathrm{~s}$. Si se acepta que la duración del EM y la duración del IEE son dos variables que controlan los índices de precisión del responder discriminado y que una mayor duración de alguna de estas variables tendría como efecto un más elevado índice de precisión, en el presente estudio no tendrían que haberse observado diferencias en los índices de precisión debido a que en las cuatro condiciones la duración del EM siempre fue de $4 \mathrm{~s}$ y el IEE se mantuvo en $12 \mathrm{~s}$. El aspecto a destacar de los resultados del presente estudio es que a pesar de mantener entre condiciones la misma duración del EM y del IEE se encontraron diferencias en los índices de precisión y que éstos siguen un orden de menor a mayor en función de la duración del intervalo entre reforzadores, en particular durante las primeras 20 sesiones de adquisición y con excepción de los sujetos de las condiciones con IER 38 s y 76 s, particularmente durante las últimas 20 sesiones, entre los que no se encontraron diferencias significativas.

Wilkie y Spetch (1978) evaluaron los efectos de imponer diferentes requisitos de respuesta durante el EM sobre el porcentaje de respuestas correctas, ellos emplearon programas de razón fija (RF) con valores de 2 y 4 respuestas. Un programa de RF4 implica una mayor duración del EM en comparación con un programa de RF2 por el tiempo que se requiere en completar el requisito de respuesta. Wilkie y Spetch reportaron que con el RF4 se observaron porcentajes de respuestas correctas más elevados que con el RF 2. Estos resultados replican los hallazgos reportados por Ferster (1960); Blough (1959); Roberts (1972); Sacks et. al. (1972) y Roberts y Grant (1976) de que el imponer diferentes requisitos de respuesta durante el EM facilita la adquisición y la precisión del responder discriminado en tareas de igualación a la muestra. Este efecto ha sido analizado en términos de que incrementar el requisito de respuesta del programa de RF alarga el tiempo de exposición del EM y con ello se contribuye a una mayor velocidad de adquisición y a más elevados índices de precisión. 
Nevin, Cumming y Berryman (1963) evaluaron los efectos del reforzamiento intermitente de las respuestas correctas, empleando programas de RF 3 , RF 6 y RF 9 en un procedimiento de igualación a la muestra; reportaron que el porcentaje de respuestas correctas incrementó en función del requisito de respuesta empleado, es decir, los mayores porcentajes de respuestas correctas se observaron en las condiciones con RF 6 y con RF9. Resultados similares fueron reportados por Mintz, Mourer y Weinberger (1966) al comparar la frecuencia de respuestas correctas bajo condiciones de RF 2 y RF 9.

Empleando una tarea de igualación a la muestra demorada, Brown y White (2005) reportaron un mayor porcentaje de respuestas correctas cuando éstas fueron reforzadas con una probabilidad de 1.0 que cuando fueron reforzadas con una probabilidad de 0.2. Este resultado no es consistente con lo observado en el presente experimento, sin embargo existen varios aspectos de procedimiento que pueden aclarar las diferencias entre los resultados. En el procedimiento de Brown y White se podía presentar como EM una luz de color rojo o de color verde, a cuyo término se presentaba una señal correlacionada con cada valor de probabilidad, una línea vertical señalaba la probabilidad de 1.0 , mientras que una cruz señalaba la probabilidad de 0.2 , inmediatamente después iniciaba el intervalo de demora EM-ECO que podía ser igual a 1 , 3, 612 ó $18 \mathrm{~s}$, las respuestas fueron reforzadas con base en los valores de probabilidad siempre y cuando el color del ECO fuera igual al del EM. Este procedimiento difiere del empleado en el presente experimento, en el estudio de Brown y White (2005) las probabilidades de reforzamiento se variaron intrasesión, se presentó una señal correlacionada con cada valor de probabilidad de reforzamiento y se emplearon diferentes duraciones del intervalo EM-ECO (i.e tarea de igualación a la muestra demorada); mientras que en el presente experimento la probabilidad de reforzamiento se varió entre condiciones, no se presentó ninguna señal correlacionada diferencialmente con la probabilidad de reforzamiento y la tarea que se empleó fue de igualación a la muestra con demora cero. Estas diferencias, en particular las relativas al señalamiento diferencial de la probabilidad de reforzamiento y la manera de variar la probabilidad de reforzamiento (intrasesión vs entre condiciones) pueden hacer la diferencia en los resultados. La presentación de la línea vertical o de la cruz "predecían" que siempre o que eventualmente las respuestas correctas fueran seguidas por el reforzador independientemente del EM presentado en cada ensayo. Si bien es cierto que la menor probabilidad de reforzamiento (0.2) resultó en un mayor espaciamiento entre reforzadores, es posible que incrementar la duración del intervalo EM-ECO modifique los efectos de la duración del intervalo entre reforzadores encontrados en el presente estudio.

Los resultados del presente estudio amplían la generalidad del efecto reportado por Flores y Bruner (2005) de que una mayor duración del IER resulta en más elevados índices de precisión y confirman que el IER es una 
variable que se confunde en los procedimientos empleados para evaluar tanto los efectos de la duración del EM como del IEE.

\section{REFERENCIAS}

Blough, D.S. (1959). Delayed matching in the pigeon. Journal of the Experimental Analysis of Behavior. 2, 151-160.

Brown, P.L. \& Jenkins, H.M. (1968). Auto-shaping of the pigeon's key-peck. Journal of the Experimental Analysis of Behavior. 11, 1-8.

Brown, G.S. \& White, G. (2005). On the effects of signaling reinforcer probability and magnitude in delayed matching to sample. Journal of the Experimental Analysis of Behavior. 83, 119-128.

DeLong, R.E., \& Wasserman, E. (1981). Effects of differential reinforcement expectancies on succesive matching-to-sample performance in pigeons. Journal of Experimental Psychology: Animal Behavior Processes. 7, 394-412.

Ferster, C. (1960). Intermittent reinforcement of matching to sample in the pigeon. Journal of the Experimental Analysis of Behavior. 3, 259-272.

Flores, C. y Bruner, C. (2005). Efecto del intervalo entre reforzadores sobre el índice de discriminación en tareas de igualación a la muestra. Acta Comportamentalia. 13, 87-97.

Goeters, S., Blakely, E., \& Poling, A. (1992). The differential outcome effect. The Psychological Record. 42, 389-411.

Holt, G. \& Shafer, J. (1973). Function of intertrial interval in matching to sample. Journal of the Experimental Analysis of Behavior. 19, 181-186.

Maki, W.S., Moe, J.C. \& Bierley, C.M. (1977). Short-term memory for stimuli, responses, and reinforcers. Journal of Experimental Psychology: Animal Behavior Processes. 3, 156-177.

Mintz. D.E., Mourer, D.J. \& Weinberger, L. (1966). Stimulus control in fixed ratio matching to sample. Journal of the Experimental Analysis of Behavior, 9, 627-630.

Nelson, K. \& Wasserman, E. (1978). Temporal factors influencing the pigeon's successive matching-to-sample performance: sample duration, intertrial interval and retention interval. Journal of the Experimental Analysis of Behavior. 30, 153-162.

Nevin, J.A., Cumming, W.W. \& Berryman, R. (1963). Ratio reinforcement of matching behavior. Journal of the Experimental Analysis of Behavior, 6, 149-154.

Roberts, W.A. (1972). Short term memory in the pigeon: Effect of repetition and spacing. Journal of Experimental Psychology: Animal Behavior Processes. 6, 217-237.

Roberts, W.A. (1980). Distribution of trials and intertrial retention in delayed matching to sample with pigeons. Journal of Experimental Psychology: Animal Behavior Processes. 6, 217-237.

Roberts, W.A. \& Grant, D.S. (1974). Studies of short-term memory in the pigeon with presentation time precisely controlled. Learning and Motivation. 5, 393-408.

Roberts, W.A. \& Grant, D.S. (1976). Studies of short-term memory in the pigeon using the delayed matching to sample procedure. En D. Medin, W. Roberts y R. Davis (Eds.). Processes of animal memory. Hillsdale, New Jersey: Lawrence Erlbaum. 
Roberts, W.A. \& Kraemer, P.J. (1982). Some observations of the effects of intertrial interval and delay on delayed matching to sample in pigeons. Journal of Experimental Psychology: Animal Behavior Processes. 8, 342-353.

Sacks, R.A., Kamil, A.C \& Mack, R. (1972). The effects of fixed-ratio sample requirements on matching to sample in the pigeon. Psychonomic Science, 26, 291-293.

Santi, A. \& Roberts, W.A. (1985a). Prospective representaction: The effects of varied mapping of sample stimuli to comparison stimuli and differential trial outcomes on pigeons' working memory. Animal Learning and Behavior. 13, 103-108

Santi, A. \& Roberts, W.A (1985b). Reinforcement expectancy and trial spacing effects in delayed matching-to-sample by pigeons. Animal Learning and Behavior. 13, 274-284.

Urcuioli, P.J. (1990a). Differential outcomes and many-to-one matching: Effects of correlation with correct choice. Animal Learning and Behavior. 18, 410-422.

Urcuioli, P.J. (1990b). Some relationships between outcome expectancies and sample stimuli in pigeons' delayed matching. Animal Learning and Behavior. 18, 302314.

Wilkie, D.M. \& Spetch, M.L. (1978). The effect of sample and comparison ratio schedules on delayed matching to sample in the pigeon. Animal Learning \& Behavior. 6, 273-278. 